\title{
The complementary extended Weibull power series class of distributions
}

\section{A classe complementar de distribuições Weibull estendida séries de potência}

\author{
Gauss M. Cordeiro*1 and Rodrigo B. Silva ${ }^{+1}$ \\ ${ }^{1}$ Departamento de Estatística, Centro de Ciências Exatas e da Natureza, \\ Universidade Federal de Pernambuco
}

\begin{abstract}
In this paper, we introduce a general method for obtaining more flexible new distributions by compounding the extended Weibull and power series distributions on a latent complementary risk problem base. The properties of the proposed class are discussed, including a formal proof of an expension for its density function and explicit formulae for its hazard rate function, quantiles, ordinary and incomplete moments and generating function. The method of maximum likelihood is used for estimating the model parameters. Special distributions are investigated. The potentiality of the new class is illustrated on a real data set.
\end{abstract}

Keywords: Extended Weibull distribution, Extended Weibull power series distribution, Maximum likelihood, Power series distribution.

\section{Resumo}

Neste artigo, introduzimos um método geral para obter distribuições de probabilidade mais flexíveis por meio da composição das classes Weibull estendida e da séries de potência no contexto de riscos competitivos. As propriedades da nova classe são descritas, incluindo uma prova formal para a expansão da função densidade e fórmulas explícitas para a função taxa de risco, quantils, momentos ordinários e incompletos e função geradora. O método de máxima verossimilhança é usada para estimar os parâmetros do modelo. Algumas distribuições especiais são estudadas. A potencialidade da nova classe é ilustrada com uma aplicação a um conjunto de dados reais.

Palavras-chave: Distribuição Weibull estendida, distribuição Weibull estendida séries de potência, máxima verossimilhança, distribuição de séries de potência.

\footnotetext{
*Corresponding author: gauss@de.ufpe.br

${ }^{\dagger}$ R. B. Silva: rodrigobs29@gmail.com

Received: 01/16/2014 Reviewed: 05/20/2014
} 


\section{Introduction}

$\mathrm{T}$ He complementary risk problems arises in several areas, such as public health, actuarial science, biomedical studies, demography and industrial reliability. In complementary risk scenarios, the event of interest is related to causes which are not completely observed. Therefore, the lifetime of the event of interest is modelled as function of the available information, which is only the maximum ordered lifetime value among all causes. For example, in medical applications, the death of a patient can be caused by several competing causes such as heart failure, pulmonary embolism and stroke. Further, in industrial applications, the failure of a device can be caused by several competing causes such as the failure of a component, contamination from dirt, an assembly error, harsh working environments, among others. For more literature on complementary risk problems, we refer the reader to Cox and Oakes (1984), Crowder et al. (1991), Louzada-Neto (1999) and Lawless (2003).

In recent years, several compound models have been introduced by complementary risk motivation. For instance, Bereta et al. (2010) proposed the Weibull Poisson distribution, which can be used for modeling the lifetime until the occurrence of a failure in competing risks scenarios. In the same context, Barriga et al. (2011), Louzada et al. (2011) and Flores et al. (2011) defined the complementary exponential power (CEP), complementary exponential geometric (CEG) and complementary exponential power series (CEPS) distributions, respectively. More recently, Louzada et al. (2013) proposed the complementary exponentiated exponential geometric (CEEG) distribution.

On the other hand, Silva et al. (2013) defined general models for the structure of primary causes based on extended types of failure of a system, called the extended Weibull power series (EWPS) distributions. The EWPS models are obtained by compounding the extended Weibull (EW) (Gurvich et al., 1997) and power series distributions. The compounding procedure follows the same set-up pioneered by Marshall and Olkin (1997) and defines 68 special models. The EWPS class is derived as follows. First, consider the EW class of distributions with cumulative distribution function (cdf) given by

$$
G(x ; \alpha, \xi)=1-\mathrm{e}^{-\alpha H(x ; \xi)}, \quad x>0, \alpha>0,
$$

where $H(x ; \xi)$ is a non-negative monotonically increasing function which depends on a parameter vector $\xi$. The corresponding probability density function (pdf) becomes

$$
g(x ; \alpha, \boldsymbol{\xi})=\alpha h(x ; \xi) \mathrm{e}^{-\alpha H(x ; \xi)}, \quad x>0, \alpha>0,
$$

where $h(x ; \xi)$ is the first derivative of $H(x ; \xi)$. We emphasize that several distributions could be expressed in the form (1). Table 1 summarizes several of these models. Further, we refer the reader to Nadarajah and Kotz (2005) and Pham and Lai (2007). Second, let N be a discrete random variable having a power series distribution (truncated at zero) with probability mass function

$$
P(N=n)=\frac{a_{n} \theta^{n}}{C(\theta)}, n=1,2, \ldots,
$$

where $a_{n}$ depends only on $n, C(\theta)=\sum_{n=1}^{\infty} a_{n} \theta^{n}$ (with $\theta>0)$ is such that $C(\theta)$ is finite. Table 2 summarizes some power series distributions (truncated at zero) defined according to (3) such as the Poisson, logarithmic, geometric and binomial distributions. Given $N$, let $X_{1}, \ldots, X_{N}$ be independent and identically distributed (iid) random variables following (1). The EWPS class is defined by the random variable $X=\min \left\{X_{i}\right\}_{i=1}^{N}$, which has the cdf given by

$$
F(x ; \theta, \alpha, \xi)=1-\frac{C\left(\theta \mathrm{e}^{-\alpha H(x ; \xi)}\right)}{C(\theta)}, \quad x>0 .
$$

However, the EWPS models are not suitable for modeling the observed maximum lifetime among all causes. For this purpose, we define the complementary extended Weibull power series (CEWPS) class of univariate distributions obtained by compounding the extended Weibull and power series distributions. The compounding procedure follows the same way of Barriga et al. (2011) and Flores et al. (2011).

This paper is organized as follows. In Section 2, we define the CEWPS class of distributions and demonstrate that there are many existing models which can be derived as special cases of the proposed unified model. In Section 3, we provide general properties of the CEWPS class including the density, survival and hazard rate functions, and some useful expansions for the quantiles, ordinary and incomplete moments and generating function. Estimation of the parameters by maximum likelihood is discussed in Section 4. Two special models of the proposed class are investigated in Section 5. Applications to a real data set is presented in Section 6. Some concluding remarks are addressed in Section 7.

\section{The new class}

The new class of distributions can be derived as follows. Let $N$ be a discrete random variable having a power series distribution (3). Given $N$, let $X_{1}, \ldots, X_{N}$ be iid random variables following $(1)$. Let $X_{(n)}=\max \left\{X_{i}\right\}_{i=1}^{N}$. The conditional cumulative distribution of $X_{(n)} \mid N=n$ is given by

$$
G_{X_{(n)} \mid N=n}(x)=\left[1-\mathrm{e}^{-\alpha H(x ; \xi)}\right]^{n},
$$


Table 1: Special distributions and corresponding $H(x ; \xi)$ and $h(x ; \xi)$ functions

\begin{tabular}{|c|c|c|c|c|c|}
\hline Distribution & $H(x ; \xi)$ & $h(x ; \xi)$ & $\alpha$ & $\xi$ & References \\
\hline Exponential $(x \geq 0)$ & $x$ & 1 & $\alpha$ & $\varnothing$ & Johnson et al. (1994) \\
\hline Pareto $(x \geq k)$ & $\log (x / k)$ & $1 / x$ & $\alpha$ & $k$ & Johnson et al. (1994) \\
\hline Rayleigh $(x \geq 0)$ & $x^{2}$ & $2 x$ & $\alpha$ & $\varnothing$ & Rayleigh (1880) \\
\hline Weibull $(x \geq 0)$ & $x^{\gamma}$ & $\gamma x^{\gamma-1}$ & $\alpha$ & $\gamma$ & Johnson et al. (1994) \\
\hline Modified Weibull $(x \geq 0)$ & $x^{\gamma} \exp (\lambda x)$ & $x^{\gamma-1} \exp (\lambda x)(\gamma+\lambda x)$ & $\alpha$ & {$[\gamma, \lambda]$} & Lai et al. (2003) \\
\hline Weibull extension $(x \geq 0)$ & $\lambda\left[\exp (x / \lambda)^{\beta}-1\right]$ & $\beta \exp (x / \lambda)^{\beta}(x / \lambda)^{\beta-1}$ & $\alpha$ & {$[\gamma, \lambda, \beta]$} & Xie et al. (2002) \\
\hline Log-Weibull $(-\infty<x<\infty)$ & $\exp [(x-\mu) / \sigma]$ & $(1 / \sigma) \exp [(x-\mu) / \sigma]$ & 1 & {$[\mu, \sigma]$} & White (1969) \\
\hline Phani $(0<\mu<x<\sigma<\infty)$ & {$[(x-\mu) /(\sigma-x)]^{\beta}$} & $\beta[(x-\mu) /(\sigma-x)]^{\beta-1}\left[(\sigma-\mu) /(\sigma-t)^{2}\right]$ & $\alpha$ & {$[\mu, \sigma, \beta]$} & Phani (1987) \\
\hline Weibull Kies $(0<\mu<x<\sigma<\infty)$ & $(x-\mu)^{\beta_{1}} /(\sigma-x)^{\beta_{2}}$ & $(x-\mu)^{\beta_{1}-1}(\sigma-x)^{-\beta_{2}-1}\left[\beta_{1}(\sigma-x)+\beta_{2}(x-\mu)\right]$ & $\alpha$ & {$\left[\mu, \sigma, \beta_{1}, \beta_{2}\right]$} & Kies (1958) \\
\hline Additive Weibull $(x \geq 0)$ & $\left(x / \beta_{1}\right)^{\alpha_{1}}+\left(x / \beta_{2}\right)^{\alpha_{2}}$ & $2\left(\alpha_{1} / \beta_{1}\right)\left(x / \beta_{1}\right)^{\alpha_{1}-1}+\left(\alpha_{2} / \beta_{2}\right)\left(x / \beta_{2}\right)^{\alpha_{2}-1}$ & 1 & {$\left[\alpha_{1}, \alpha_{2}, \beta_{1}, \beta_{2}\right]$} & Xie and Lai (1995) \\
\hline Traditional Weibull $(x \geq 0)$ & $x^{b}\left[\exp \left(c x^{d}-1\right)\right]$ & $b x^{b-1}\left[\exp \left(c x^{d}\right)-1\right]+c d x^{b+d-1} \exp \left(c x^{d}\right)$ & $\alpha$ & {$[b, c, d]$} & Nadarajah and Kotz (2005) \\
\hline Gen. power Weibull $(x \geq 0)$ & {$\left[1+(x / \beta)^{\alpha_{1}}\right]^{\theta}-1$} & $\left(\theta \alpha_{1} / \beta\right)\left[1+(x / \beta)^{\alpha_{1}}\right]^{\theta-1}(x / \beta)^{\alpha_{1}}$ & 1 & {$\left[\alpha_{1}, \beta, \theta\right]$} & Nikulin and Haghighi (2006) \\
\hline Flexible Weibull extension $(x \geq 0)$ & $\exp \left(\alpha_{1} x-\beta / x\right)$ & $\exp \left(\alpha_{1} x-\beta / x\right)\left(\alpha_{1}+\beta / x^{2}\right)$ & 1 & {$\left[\alpha_{1}, \beta\right]$} & Bebbington et al. (2007) \\
\hline Gompertz $(x \geq 0)$ & $\beta^{-1}[\exp (\beta x)-1]$ & $\exp (\beta x)$ & $\alpha$ & $\beta$ & Gompertz (1825) \\
\hline Exponential power $(x \geq 0)$ & $\exp \left[(\lambda x)^{\beta}\right]-1$ & $\beta \lambda \exp \left[(\lambda x)^{\beta}\right](\lambda x)^{\beta-1}$ & 1 & {$[\lambda, \beta]$} & Smith and Bain (1975) \\
\hline Chen $(x \geq 0)$ & $\exp \left(x^{b}\right)-1$ & $b x^{b-1} \exp \left(x^{b}\right)$ & $\alpha$ & $b$ & Chen (2000) \\
\hline Pham $(x \geq 0)$ & $\left(a^{x}\right)^{\beta}-1$ & $\beta\left(a^{x}\right)^{\beta} \log (a)$ & 1 & {$[a, \beta]$} & Pham (2002) \\
\hline
\end{tabular}

Table 2: Useful quantities for some power series distributions

\begin{tabular}{lllllll}
\hline Distribution & $a_{n}$ & $C(\theta)$ & $C^{\prime}(\theta)$ & $C^{\prime \prime}(\theta)$ & $C(\theta)^{-1}$ & $\Theta$ \\
\hline Poisson & $n !^{-1}$ & $\mathrm{e}^{\theta}-1$ & $e^{\theta}$ & $e^{\theta}$ & $\log (\theta+1)$ & $\theta \in(0, \infty)$ \\
Logarithmic & $n^{-1}$ & $-\log (1-\theta)$ & $(1-\theta)^{-1}$ & $(1-\theta)^{-2}$ & $1-\mathrm{e}^{-\theta}$ & $\theta \in(0,1)$ \\
Geometric & 1 & $\theta(1-\theta)^{-1}$ & $(1-\theta)^{-2}$ & $2(1-\theta)^{-3}$ & $\theta(\theta+1)^{-1}$ & $\theta \in(0,1)$ \\
Binomial & $\left(\begin{array}{c}m \\
n\end{array}\right)$ & $(\theta+1)^{m}-1$ & $m(\theta+1)^{m-1}$ & $\frac{m(m-1)}{(\theta+1)^{2-m}}$ & $(\theta-1)^{1 / m}-1$ & $\theta \in(0,1)$ \\
\hline
\end{tabular}

i.e., $X_{(n)} \mid N=n$ has the exponentiated form of the general class (1) with parameters $n, \alpha$ and $\xi$ based on the same $H(x ; \xi)$ function. Thus, we obtain

$$
P\left(X_{(n)} \leq x, N=n\right)=\frac{a_{n} \theta^{n}}{C(\theta)}\left[1-\mathrm{e}^{-\alpha H(x ; \xi)}\right]^{n}, x>0, n \geq 1 \text {. }
$$

So, the EWPS class of distributions is defined by the marginal cdf of $X_{(n)}$ :

$$
F(x ; \theta, \alpha, \xi)=\frac{C\left[\theta\left(1-\mathrm{e}^{-\alpha H(x ; \xi)}\right)\right]}{C(\theta)}, \quad x>0 .
$$

We can provide at least two motivations for the CEWPS class. For instance, from the stochastic representation $X=\max \left\{X_{i}\right\}_{i=1}^{N}$, we note that the CEWPS class can arise in parallel systems with identical components, which appear in many industrial applications and biological organisms. Further, under the so-called last-activation scheme, let $N$ be the number of latent factors that must all be active by failure and $X_{i}$ be the time of resistance to a disease manifestation due to the $i$ th latent factor. In the last-activation scheme, it is assumed that failure occurs after all $N$ factors have been active. So, if the $X_{i}$ 's are iid EW random variables independent of $N$, where $N$ follows a zero-truncated power series distribution, the CEWPS class can be able for modeling the time to the failure under the last-activation scheme.

Hereafter, the random variable $X$ following (4) with parameters $\theta, \alpha$ and the vector of parameters $\xi$ is denoted by $X \sim \operatorname{CEWPS}(\theta, \alpha, \xi)$. Equation (4) extends 
some distributions which have been studied in the literature. The complementary exponential geometric (CEG) distribution is obtained by taking $H(x ; \xi)=x$ and $C(\theta)=\theta(1-\theta)^{-1}$ with $\theta \in(0,1)$. Further, from $H(x ; \xi)=x$ and $C(\theta)=\mathrm{e}^{\theta}-1, \theta>0$, we obtain the complementary Poisson exponential (CPE) distribution proposed by Cancho et al. (2011). The complementary exponential power series (CEPS) distributions come from (4) by combining $H(x ; \xi)=x$ with any $C(\theta)$ listed in Table 2

\section{General properties}

\subsection{Density, survival and hazard rate func- tions}

The density function associated to 4 is given by

$f(x ; \theta, \alpha, \xi)=\theta \alpha h(x ; \xi) \mathrm{e}^{-\alpha H(x ; \xi)} \frac{C^{\prime}\left[\theta\left(1-\mathrm{e}^{-\alpha H(x ; \xi)}\right)\right]}{C(\theta)}, x>0$.

We can obtain the EW class of distributions as a limiting special case of the CEWPS class of distributions when $\theta \rightarrow 0^{+}$. Indeed, using a similar argument given by Morais and Barreto-Souza (2011), we have (for $x>0$ )

$$
\begin{aligned}
& \lim _{\theta \rightarrow 0^{+}} F(x)=\lim _{\theta \rightarrow 0^{+}} \frac{\sum_{n=1}^{\infty} a_{n} \theta^{n}\left[1-\mathrm{e}^{-\alpha H(x ; \xi)}\right]^{n}}{\sum_{n=1}^{\infty} a_{n} \theta^{n}} \\
& =\lim _{\theta \rightarrow 0^{+}} \frac{1-\mathrm{e}^{-\alpha H(x ; \xi)}+a_{1}^{-1} \sum_{n=2}^{\infty} a_{n} \theta^{n-1}\left[1-\mathrm{e}^{-\alpha H(x ; \xi)}\right]^{n}}{1+a_{1}^{-1} \sum_{n=2}^{\infty} a_{n} \theta^{n-1}} \\
& =1-\mathrm{e}^{-\alpha H(x ; \xi)} .
\end{aligned}
$$

For instance, for $H(x ; \xi)=\exp \left[(\lambda x)^{\beta}\right]-1$ and $\theta \rightarrow$ $0^{+}$, we obtain the exponential-power distribution, which was defined by Smith and Bain (1975). Moreover, we provide an interesting expansion for the density (5). We have $C^{\prime}(\theta)=\sum_{n=1}^{\infty} n a_{n} \theta^{n-1}$. By using this result in (5), we can write

$$
f(x ; \theta, \alpha, \boldsymbol{\xi})=\sum_{n=1}^{\infty} \sum_{j=0}^{n-1} \omega_{n, j}(\theta) g(x ;(j+1) \alpha, \boldsymbol{\xi}),
$$

where

$$
\omega_{n, j}(\theta)=\frac{(-1)^{j} \theta^{n} a_{n}}{(j+1) C(\theta)}\left(\begin{array}{c}
n-1 \\
j
\end{array}\right)
$$

and $g(x ;(j+1) \alpha, \xi)$ is given by (2). Hence, the CEWPS density function is an infinite linear combination of EW densities. So, some mathematical quantities (such as the ordinary and incomplete moments, generating function and mean deviations) of the CEWPS distribution can be obtained by knowing those quantities for the baseline density function $g(x ;(j+1) \alpha, \xi)$. The CEWPS survival function becomes

$$
S(x ; \theta, \alpha, \xi)=1-\frac{C\left[\theta\left(1-\mathrm{e}^{-\alpha H(x ; \xi)}\right)\right]}{C(\theta)}
$$

and the corresponding hazard rate function (hrf) reduces to

$$
\tau(x ; \theta, \alpha, \xi)=\theta \alpha h(x ; \xi) \mathrm{e}^{-\alpha H(x ; \xi)} \frac{C^{\prime}\left[\theta\left(1-\mathrm{e}^{-\alpha H(x ; \xi)}\right)\right]}{C(\theta)-C\left[\theta\left(1-\mathrm{e}^{-\alpha H(x ; \xi)}\right)\right]} .
$$

\subsection{Quantiles, moments and generating func- tion}

The CEWPS class is easily simulated by inverting (4). The quantile function (qf) corresponding to (2) plays an important role in the algebraic developments in this section. We have

$$
Q(u ; \alpha, \xi)=H^{-1}\left\{-\frac{1}{\alpha} \log (1-u) ; \xi\right\} .
$$

We only require the inverse of $H(x ; \xi)$ to obtain the qf (7). So, if $U$ has a uniform $U(0,1)$ distribution, the solution of the nonlinear equation

$$
X=H^{-1}\left\{-\frac{1}{\alpha} \log \left[1-\frac{C^{-1}(C(\theta) U)}{\theta}\right] ; \xi\right\}
$$

has the $\operatorname{CEWPS}(\theta, \alpha, \xi)$ distribution, where $H^{-1}(\cdot)$ and $C^{-1}(\cdot)$ denote the inverse functions of $H(\cdot)$ and $C(\cdot)$, respectively. To simulate data from this nonlinear equation, we can use the matrix programming language $0 x$ through SolvenLE subroutine (see Doornik, 2007). Table 3 provides closed-form inverses for $H(x ; \xi)$ for some special models.

\subsection{Moments}

The need for necessity and the importance of moments in any statistical analysis especially in applied work is obvious. Some of the most important features and characteristics of a distribution can be studied through moments (e.g. tendency, dispersion, skewness and kurtosis).

The $r$ th raw moment of $X$ can be determined from (6) and the monotone convergence theorem. So, for $r \in \mathbb{N}$, we obtain

$$
\mathrm{E}\left(X^{r}\right)=\sum_{n=1}^{\infty} \sum_{j=0}^{n-1} \omega_{n, j}(\theta) \mathrm{E}\left(Z_{j+1}^{r}\right)
$$

where $Z_{j+1}$ denotes a random variable with density function $g(z ;(j+1) \alpha, \xi)$. 
Table 3: Inverse function $x=H^{-1}(x ; \xi)$ for some EW models

\begin{tabular}{lll}
\hline Distribution & $z=H^{-1}(x ; \xi)$ & References \\
\hline Exponential power & $\lambda^{-1}[\log (x+1)]^{1 / \beta}$ & Smith and Bain (1975) \\
Chen & {$[\log (x+1)]^{1 / \beta}$} & Chen (2000) \\
Log-Weibull & $\sigma \log (x)+\mu$ & White (1969) \\
Weibull Kies & $\left(t^{1 / \beta} \sigma+\mu\right) /\left(x^{1 / \beta}+1\right)$ & Kies (1958) \\
Generalized power Weibull & $\beta\left[(x+1)^{1 / \theta}-1\right]^{1 / \alpha_{1}}$ & Nikulin and Haghighi (2006) \\
Gompertz & $\beta^{-1} \log (\beta x+1)$ & Gompertz (1825) \\
Pham & {$\left[\log (1+x) / \log \left(a_{1}\right)\right]^{1 / \beta}$} & Pham (2002) \\
\hline
\end{tabular}

Let $\beta_{r}(\alpha, \mathcal{\xi})$ be the $r$ th moment of the EW distribution having density (2) with parameters $\alpha$ and $\xi$. We have immediately by substituting $u=G(x ; \alpha, \xi)$

$$
\beta_{r}(\alpha, \xi)=\int_{0}^{1} Q(u ; \alpha, \xi)^{r} d u
$$

Thus, we can rewrite 9 as

$$
\mathrm{E}\left(X^{r}\right)=\sum_{n=1}^{\infty} \sum_{j=0}^{n-1} \omega_{n, j}(\theta) \beta_{r}((j+1) \alpha, \boldsymbol{\xi}) .
$$

We now provide a simple application of equation (10). Consider the log-Weibull distribution for which 10) yields

$$
\beta_{s}(\alpha, \xi)=\int_{0}^{1}\{\sigma \log [-\log (1-u)]+\mu\}^{s} d u .
$$

Using the binomial expansion and setting $v=1-u$, we obtain

$$
\beta_{r}(\alpha, \boldsymbol{\xi})=\sum_{k=0}^{r}\left(\begin{array}{l}
r \\
k
\end{array}\right) \mu^{s-k} \sigma^{k} \int_{0}^{1} \log ^{k}\{-\log (v)\} d v .
$$

We can calculate this integral in MAPLE for a given $k$. For $r=1$ and $r=2$, we obtain: $\beta_{1}(\alpha, \xi)=\mu-\sigma \gamma$ and $\beta_{2}(\alpha, \xi)=\mu^{2}-2 \mu \sigma \gamma+\sigma^{2}\left[(1 / 6) \pi^{2}+\gamma^{2}\right]$, respectively, where $\gamma$ is Euler's constant.

The incomplete moments of $X$ can be determined from (6) using the monotone convergence theorem as

$$
I_{X}(r, y ; \alpha, \boldsymbol{\xi})=\int_{0}^{y} x^{r} f(x) d x=\sum_{n=1}^{\infty} \sum_{j=0}^{n-1} \omega_{n, j}(\theta) I_{Z_{j+1}}(r, y ; \alpha, \boldsymbol{\xi}),
$$

where

$$
\begin{aligned}
I_{Z_{j+1}}(r, y ; \alpha, \xi) & =\int_{0}^{y} x^{r} g(x ;(j+1) \alpha, \xi) d x \\
& =\int_{0}^{G(y ; \alpha, \xi)} Q(u ; \alpha, \xi)^{r} d u
\end{aligned}
$$

can be computed numerically for most EW distributions.

A practical application of the first incomplete moments of $X$ in equation (12) is related to the Bonferroni and Lorenz curves. They are defined by $B(\pi)=$ $\frac{1}{\pi \mu_{1}^{\prime}} I_{X}(1, q ; \alpha, \boldsymbol{\xi})$ and $L(\pi)=\frac{1}{\mu_{1}^{\prime}} I_{X}(1, q ; \alpha, \boldsymbol{\xi})$, respectively, where $\mu_{1}^{\prime}=E(X), \pi$ is a probability and

$$
q=H^{-1}\left\{-\frac{1}{\alpha} \log \left[1-\frac{C^{-1}(C(\theta) \pi)}{\theta}\right] ; \xi\right\}
$$

is determined from (8). Bonferroni and Lorenz curves have applications in economics to study income and poverty, reliability, demography, insurance and medicine.

For $r, c>0$, we define

$$
J(r, y, c)=\int_{0}^{y} x^{r} \mathrm{e}^{-c H(t ; \xi)} \mathrm{d} H(x ; \xi) .
$$

Clearly,

$$
I_{Z_{j+1}}(r, y ; \alpha, \boldsymbol{\xi})=(j+1) \alpha J(r, y,(j+1) \alpha)
$$

and then $J(r, y, c)$ becomes the basic quantity to calculate the incomplete moments of $X$.

Further, if we can invert $H(x ; \xi)$, changing variable $u=H(x ; \xi)$, equation (13) yields

$$
J(r, y, c)=\int_{H(0)}^{H(z)} H^{-1}(u)^{s} \mathrm{e}^{-u c} d u .
$$

Thus, we have two alternative forms for $J(r, y, c)$ that can be easily obtained numerically from equations (13) and (14). For some cases, we can compute $J(r, y, c)$ analytically. As an example using (14), we consider the log-Weibull distribution. We can obtain from equation 14

$$
J(r, y, c)=\int_{\exp (-\mu / \sigma)}^{\exp [(y-\mu) / \sigma]}[\sigma \log (u)+\mu]^{r} \mathrm{e}^{-u c} d u .
$$

For the first incomplete moments, $r=1$. We can calculate using MAPLE

$$
\int_{\exp (-\mu / \sigma)}^{\exp [(z-\mu) / \sigma]} \sigma \log (u) \mathrm{e}^{-u c} d u
$$




$$
\begin{aligned}
& =\frac{\sigma}{c}\left\{\exp \left(-c \mathrm{e}^{--/ œ}\right)\left(-^{-} / œ\right)+\mathrm{E}_{\mathrm{i}}\left(1, \mathrm{ce}^{-^{-/} œ}\right)\right. \\
& -\exp \left(-c \mathrm{e}^{(z-\mu) / \sigma}\right)[(z-\mu) / \sigma] \\
& \left.-E_{i}\left(1, c \mathrm{e}^{(z-\mu) / \sigma}\right)\right\},
\end{aligned}
$$

where $E_{i}(a, y)=\int_{1}^{\infty} x^{-a} \mathrm{e}^{y x} d x$ is the exponential integral. Now,

$$
\begin{aligned}
& \int_{\exp (-\mu / \sigma)}^{\exp [(z-\mu) / \sigma]} \mu \mathrm{e}^{-c u} d u \\
& =\frac{\mu}{c}\{\exp [-c \exp (-\mu / \sigma)]-\exp [-c \exp ((z-\mu) / \sigma)]\} .
\end{aligned}
$$

Then,

$$
\begin{aligned}
J(1, y, c) & =\frac{\sigma}{c}\{\exp [-c \exp (-\mu / \sigma)](-\mu / \sigma) \\
& +E_{i}(1, c \exp (-\mu / \sigma)) \\
& -\exp [-c \exp \{(z-\mu) / \sigma\}]\{(z-\mu) / \sigma\} \\
& \left.-E_{i}(1, c \exp \{(z-\mu) / \sigma\})\right\} \\
& \frac{\mu}{c}\{\exp [-c \exp (-\mu / \sigma)] \\
& -\exp [-c \exp \{(z-\mu) / \sigma\}]\} .
\end{aligned}
$$

Hence, the first incomplete moments for the complementary log-Weibull power series distribution can be determined from (12) and (13) and the last result.

We define the quantity $A(y ; \alpha, \xi)=\int_{0}^{y} x g(x ; \alpha, \xi) d x=$ $\int_{0}^{G(y ; \alpha, \xi)} Q(u ; \alpha, \xi) d u$ from the EW distribution.

We now provide a simple example for $A(y ; \alpha, \xi)$. The $\log$-Weibull distribution gives

$$
\begin{aligned}
& A(y ; \alpha, \xi) \\
& =\mu G(y ; \alpha, \xi)+\sigma \int_{0}^{G(y ; \alpha, \xi)} \log \left\{-\frac{1}{\alpha} \log (1-u)\right\} d u \\
& =\mu G(y ; \alpha, \xi)+\sigma \int_{0}^{G(y ; \alpha, \xi)} \log \{-\log (1-u)\} d u \\
& -\sigma G(y ; \alpha, \xi) \log (\alpha),
\end{aligned}
$$

where $G(y ; \alpha, \xi)=1-\exp \left\{-\exp \left(\frac{y-\mu}{\sigma}\right)\right\}$.

Setting $v=1-u$, the integral calculated using MAPLE becomes

$\int_{x}^{1} \log \{-\log (v)\} d v=-x \log [-\log (x)]-E_{i}(1,-\log (x))-\gamma$.

Then,

$$
\begin{aligned}
& A(y ; \alpha, \xi)=\mu G(y ; \alpha, \xi)-\sigma G(y ; \alpha, \xi) \log (\alpha) \\
& \quad+\sigma\{-[1-G(y ; \alpha, \xi)] \log \{-\log [1-G(y ; \alpha, \xi)]\} \\
& \left.\quad-E_{i}(1,-\log [1-G(y ; \alpha, \xi)])-\gamma\right\} .
\end{aligned}
$$

\subsection{Generating function}

The moment generating function (mgf) of a random variable provides the basis of an alternative route to analytical results compared with working directly with its pdf and cdf. The mgf of $X$ can be determined from (6) as

$$
M_{X}(t ; \alpha, \xi)=\sum_{n=1}^{\infty} \sum_{j=0}^{n-1} \omega_{n, j}(\theta) \mathrm{E}\left(\mathrm{e}^{t Z_{j+1}}\right) .
$$

The mgf of the random variable $Z_{j+1}$ can be expressed as

$$
M(t ; \alpha, \tilde{\xi})=\int_{0}^{1} \exp \{t Q(u ; \alpha, \xi)\} d u .
$$

Based on the last two equations, we can write the mgf of $X$ as

$$
M_{X}(t ; \alpha, \boldsymbol{\xi})=\sum_{n=1}^{\infty} \sum_{j=0}^{n-1} \omega_{n, j}(\theta) M(t ;(j+1) \alpha, \boldsymbol{\xi}) .
$$

We give two applications of equation (15). First, consider the log-Weibull distribution whose qf is given by

$$
Q(u ; \alpha, \tilde{\xi})=\sigma \log \left[-\frac{1}{\alpha} \log (1-u)\right]+\mu,
$$

where $\xi=\mu, \sigma$. We can readily obtain from equation (15)

$M(t ; \alpha, \xi)=\int_{0}^{1} \exp \left[t\left\{\sigma \log \left[-\frac{1}{\alpha} \log (1-u)\right]+\mu\right\}\right] d u$ and then

$$
M(t ; \alpha, \xi)=\alpha^{-t \sigma} \mathrm{e}^{t \mu} \int_{0}^{1} \log ^{t \sigma}\left(\frac{1}{1-u}\right) d u .
$$

Changing variable $v=1-u$, we can write

$$
M(t ; \alpha, \xi)=\alpha^{-t \sigma} \mathrm{e}^{t \mu} \int_{0}^{1} \log ^{t \sigma}(v) d v
$$

and then from Prudnikov et al. (1986, equation 2.6.3.1), we have

$$
M(t ; \alpha, \boldsymbol{\xi})=\Gamma(\sigma t+1) .
$$

By combining this equation with (16), we obtain the mgf of the complementary log-Weibull power series distribution.

Secondly, we consider the Gompertz distribution for which $H(x ; \beta)=\beta^{-1}[\exp (\beta x)-1]$ and $h(x ; \beta)=$ $\exp (\beta x)$, where $-\infty<\beta<\infty$. The Gompertz qf is

$$
Q(u ; \alpha, \beta)=\frac{\log \left[\left(-\alpha^{-1}\right) \log (1-u)+1\right]}{\beta} .
$$

We can readily obtain from 15

$$
M(t ; \alpha, \beta)=\int_{0}^{1} \exp \{t \log [-(\beta / \alpha) \log (1-u)+1]\} d u
$$




$$
=\int_{0}^{1}[-(\beta / \alpha) \log (1-u)+1]^{t} d u .
$$

Using the binomial expansion and changing $v=$ $1-u$, we have

$$
M(t ; \alpha, \beta)=\sum_{j=0}^{\infty}\left(\begin{array}{l}
t \\
j
\end{array}\right)\left(\frac{\beta}{\alpha}\right)^{j} \int_{0}^{1} \log ^{j}(v) d v .
$$

But $\int_{0}^{1} \log ^{j}(v) d v=(-1)^{j} \Gamma(j+1)$ and then

$$
\begin{aligned}
M(t ; \alpha, \beta) & =\left(\frac{\beta}{\alpha}\right)^{(t+1)} \exp \left(\frac{\beta}{\alpha}\right)\left\{(t+1) \Gamma\left(-t-1 ; \frac{\beta}{\alpha}\right)\right. \\
& +\Gamma(-t)\} \Gamma(t+1)^{2},
\end{aligned}
$$

where $\Gamma(x ; \alpha)=\int_{x}^{\infty} w^{\alpha-1} \mathrm{e}^{-w} d w$ is the upper incomplete gamma function.

By combining this equation with (16), we obtain the mgf of the complementary Gompertz power series distribution.

Equations (9), (11), (12), (15) and (16) are the main results of this section.

\subsection{Mean deviations}

The amount of scatter in a population is evidently measured to some extent by the totality of deviations from the mean and median. We can easily obtain the mean deviations about the ordinary mean $\mu_{1}^{\prime}=E(X)$ and about the median $M$ from equations

$$
\begin{aligned}
& \delta_{1}=2\left[\mu_{1}^{\prime} F\left(\mu_{1}^{\prime}\right)-I_{X}\left(1, \mu_{1}^{\prime} ; \alpha, \xi\right)\right] \text { and } \\
& \delta_{2}=\mu_{1}^{\prime}-2 I_{X}(1, M ; \alpha, \xi)
\end{aligned}
$$

where $I_{X}(1, q ; \alpha, \xi)$ comes from 12 with $r=1$.

\section{Maximum likelihood estimation}

Here, we determine the maximum likelihood estimates (MLEs) of the parameters of the CEWPS class of distributions from complete samples only. Let $x_{1}, \ldots, x_{n}$ be observed values from the CEWPS class with parameters $\theta, \alpha$ and $\xi$. Let $\Theta=(\theta, \alpha, \xi)^{\top}$ be the $p \times 1$ parameter vector. The total log-likelihood function for $\Theta$ is given by

$$
\begin{aligned}
\ell_{n} & =\ell_{n}(\Theta)=n[\log (\theta)+\log (\alpha)-\log (C(\theta))] \\
& -\alpha \sum_{i=1}^{n} H\left(x_{i} ; \xi\right)+\sum_{i=1}^{n} \log \left[h\left(x_{i} ; \xi\right)\right] \\
& +\sum_{i=1}^{n} \log \left\{C^{\prime}\left[\theta\left(1-\mathrm{e}^{-\alpha H(x ; \xi)}\right)\right]\right\} .
\end{aligned}
$$

The log-likelihood can be maximized either directly by using the SAS (PROC NLMIXED) or the Ox program (sub-routine MaxBFGS) (see Doornik, 2007) or by solving the nonlinear likelihood equations obtained by differentiating (17). The components of the score function $U_{n}(\Theta)=\left(\partial \ell_{n} / \partial \theta, \partial \ell_{n} / \partial \alpha, \partial \ell_{n} / \partial \xi\right)^{\top}$ are

$$
\begin{aligned}
\frac{\partial \ell_{n}}{\partial \alpha} & =\frac{n}{\alpha}-\sum_{i=1}^{n} H\left(x_{i} ; \xi\right) \\
& +\theta \sum_{i=1}^{n} H\left(x_{i} ; \xi\right) \mathrm{e}^{-\alpha H\left(x_{i} ; \xi\right)} \frac{C^{\prime \prime}\left[\theta\left(1-\mathrm{e}^{-\alpha H(x ; \xi)}\right)\right]}{C^{\prime}\left[\theta\left(1-\mathrm{e}^{-\alpha H(x ; \xi)}\right)\right]}, \\
\frac{\partial \ell_{n}}{\partial \theta} & =\frac{n}{\theta}-n \frac{C^{\prime}(\theta)}{C(\theta)} \\
& +\sum_{i=1}^{n}\left(1-\mathrm{e}^{-\alpha H\left(x_{i} ; \xi\right)}\right) \frac{C^{\prime \prime}\left[\theta\left(1-\mathrm{e}^{-\alpha H(x ; \xi)}\right)\right]}{C^{\prime}\left[\theta\left(1-\mathrm{e}^{-\alpha H(x ; \xi)}\right)\right]}
\end{aligned}
$$

and

$$
\begin{aligned}
\frac{\partial \ell_{n}}{\partial \xi_{k}} & =\sum_{i=1}^{n} \frac{\partial \log h\left(x_{i} ; \xi\right)}{\partial \xi_{k}} \\
& -\alpha \sum_{i=1}^{n} \frac{\partial H\left(x_{i} ; \xi\right)}{\partial \xi_{k}}\left[1-\theta \mathrm{e}^{-\alpha H\left(x_{i} ; \xi\right)} \frac{C^{\prime \prime}\left[\theta\left(1-\mathrm{e}^{-\alpha H(x ; \xi)}\right)\right]}{C^{\prime}\left[\theta\left(1-\mathrm{e}^{-\alpha H(x ; \xi)}\right)\right]}\right] .
\end{aligned}
$$

For interval estimation on the model parameters, we require the observed total information matrix

$$
J_{n}(\Theta)=-\left(\begin{array}{ccc}
U_{\theta \theta} & U_{\theta \alpha} & U_{\theta \xi}^{\top} \\
U_{\alpha \theta} & U_{\alpha \alpha} & U_{\alpha \xi}^{\top} \\
U_{\theta \xi} & U_{\alpha \xi} & U_{\tilde{\xi}}^{\mathcal{\zeta}}
\end{array}\right) .
$$

Let $\widehat{\Theta}$ be the MLE of $\Theta$. Under standard regular conditions (Cox and Hinkley, 1974) that are fulfilled for the proposed model whenever the parameters are in the interior of the parameter space, we can approximate the distribution of $\sqrt{n}(\widehat{\Theta}-\Theta)$ by the multivariate normal $N_{p}\left(0, K(\Theta)^{-1}\right)$, where $K(\Theta)=\lim _{n \rightarrow \infty} n^{-1} J_{n}(\Theta)$ is the unit information matrix and $p$ is the number of parameters of the compounded distribution.

\section{Special models}

\subsection{Complementary modified Weibull geo- metric distribution}

The complementary modified Weibull geometric (CMWG) distribution is defined by the cdf (5) with $H(x ; \xi)=$ $x^{\gamma} \exp (\lambda x)$ and $C(\theta)=\theta(1-\theta)^{-1}$ leading to

$$
\begin{aligned}
f(x ; \theta, \alpha, \gamma, \lambda) & =\alpha(1-\theta) x^{\gamma-1} \mathrm{e}^{\lambda x}(\gamma+\lambda x) \\
& \frac{\exp \left(-\alpha x^{\gamma} \mathrm{e}^{\lambda x}\right)}{\left\{1-\theta\left[1-\exp \left(-\alpha x \gamma \mathrm{e}^{\lambda x}\right)\right]\right\}^{2}}, \quad x>0,
\end{aligned}
$$

where $\theta \in(0,1)$. The associated hrf becomes

$$
\tau(x ; \theta, \alpha, \gamma, \lambda)=\alpha(1-\theta)^{-1} x^{\gamma-1} \mathrm{e}^{\lambda x}(\gamma+\lambda x)
$$




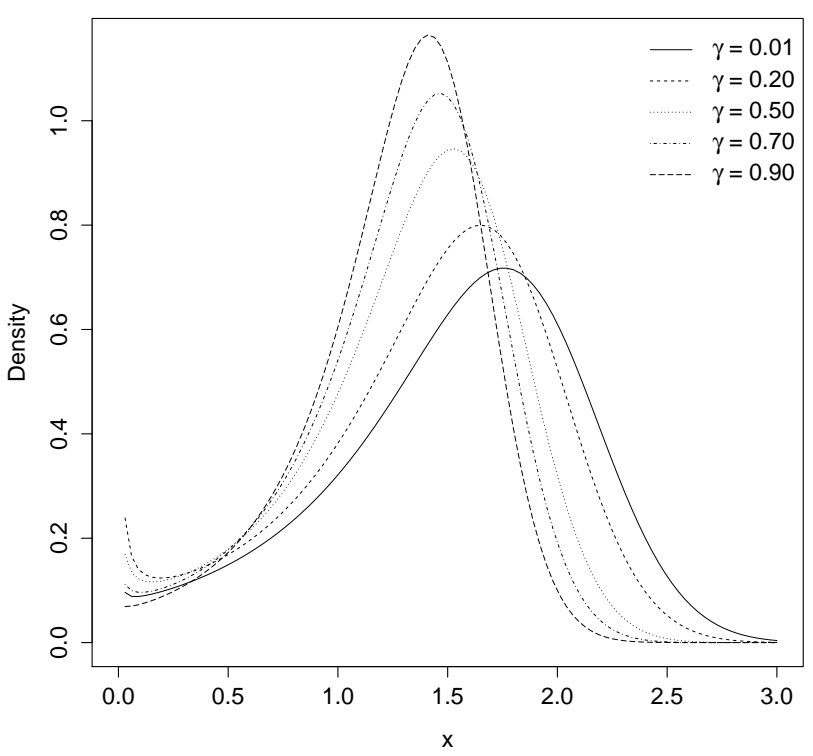

(a) $\alpha=0.5, \theta=0.2$ and $\lambda=0.6$

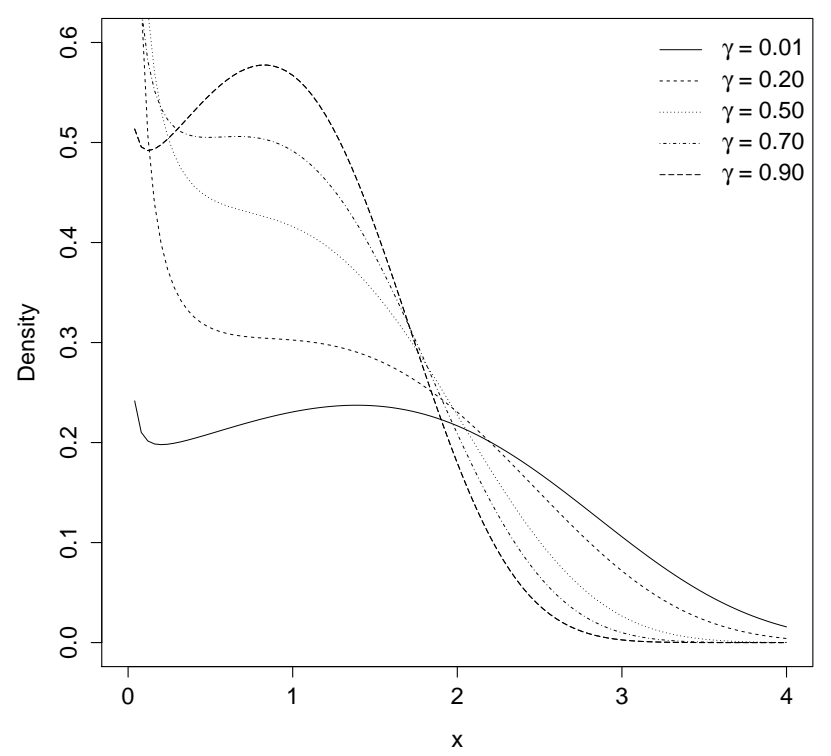

(b) $\alpha=0.5, \theta=0.9$ and $\lambda=1.0$

Figure 1: Plots of the CMWG densities for some parameter values.

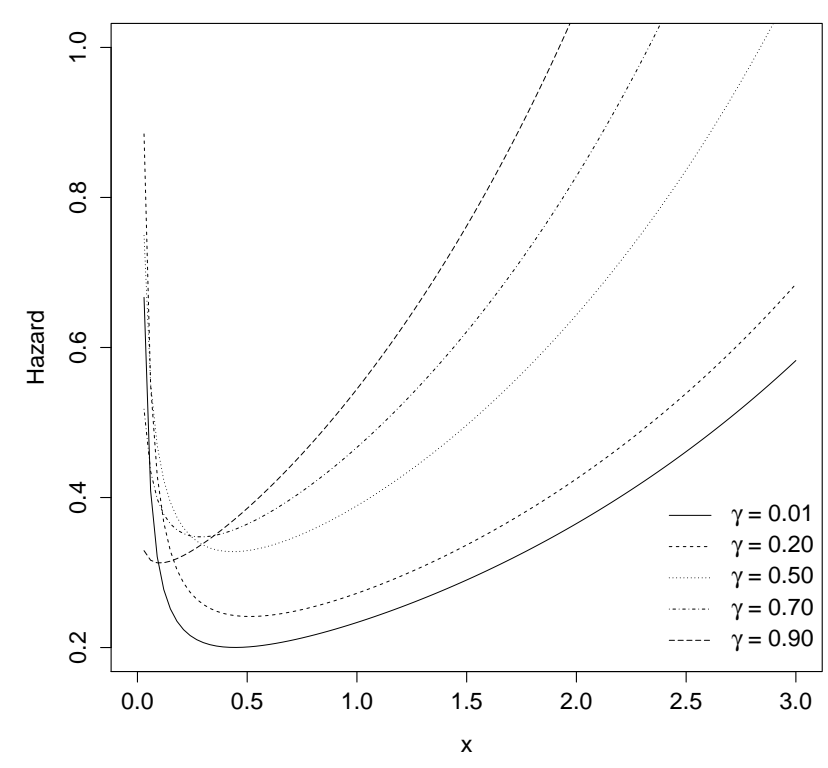

(a) $\alpha=0.2, \theta=0.2$ and $\lambda=0.5$

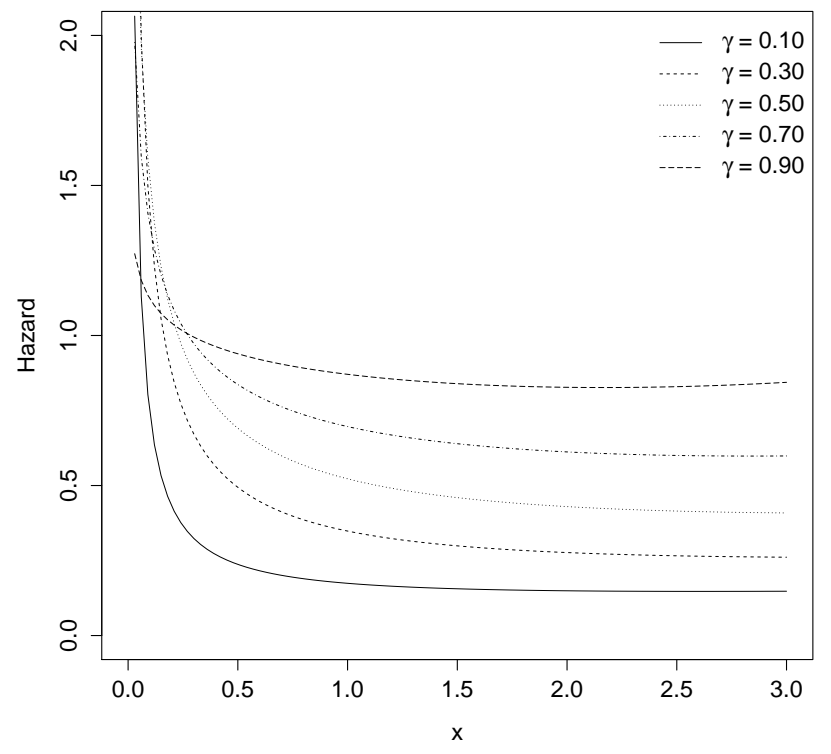

(b) $\alpha=0.5, \theta=0.5$ and $\lambda=0.1$

Figure 2: Plots of the CMWG hrfs for some parameter values.

$$
\left\{1-\theta\left[1-\exp \left(-\alpha x^{\gamma} \mathrm{e}^{\lambda x}\right)\right]\right\}, \quad x>0 .
$$

As stated before, the CMWG distribution includes the modified Weibull (MW) distribution when $\theta \rightarrow 0^{+}$. Further, for $\theta \rightarrow 0^{+}$and $\lambda=0$, we obtain the Weibull distribution. Thus, the exponential and Rayleigh distributions follow as special models of the CMWG distribution. Figures 1 and 2 display the density and hazard rate functions of the CMWG distribution for selected parameter values.

The $r$ th raw moment of the random variable $X$ having the CMWG distribution is determined in closed-form from $(6)$ as

$$
\mathrm{E}\left(X^{r}\right)=\sum_{n=1}^{\infty} \sum_{j=0}^{n-1} \omega_{n, j}(\theta) \mu_{r}(n),
$$


where $\mu_{r}(n)=\int_{0}^{\infty} x^{r} g(x ;(j+1) \alpha, \gamma, \lambda) d x$ denotes the $r$ th raw moment of the MW distribution with parameters $(j+1) \alpha, \gamma$ and $\lambda$. Carrasco et al. (2008) obtained an infinite representation for the $r$ th raw moment of the MW distribution with these parameters given by

$$
\mu_{r}(n)=\sum_{i_{1}, \ldots, i_{r}=1}^{\infty} \frac{A_{i_{1}, \ldots, i_{r}} \Gamma\left(s_{r} / \gamma+1\right)}{[(k+1) \alpha]^{s_{r} / \gamma}},
$$

where

$$
A_{i_{1}, \ldots, i_{r}}=a_{i_{1}}, \ldots, a_{i_{r}} \text { and } s_{r}=i_{1}, \ldots, i_{i}
$$

and

$$
a_{i}=\frac{(-1)^{i+1} i^{i-2}}{(i-1) !}\left(\frac{\lambda}{\gamma}\right)^{i-1} .
$$

Hence, the ordinary moments of $X$ can be obtained directly from equations (18) and (19).

\subsection{Complementary Pareto Poisson distribu- tion}

The complementary Pareto Poisson (CPP) distribution is defined by taking $H(x ; \xi)=\log (x / k)$ and $C(\theta)=\mathrm{e}^{\theta}-1$ in (4) leading to

$f(x ; \theta, \alpha, k)=\theta \alpha k^{\alpha} x^{-(\alpha+1)} \frac{\exp \left\{\theta\left[1-(k / x)^{\alpha}\right]\right\}}{\mathrm{e}^{\theta}-1}, x \geq k$.

The corresponding hrf is

$$
\tau(x ; \theta, \alpha, k)=\frac{\theta \alpha k^{\alpha} x^{-(\alpha+1)}}{\exp \left[\theta(k / x)^{\alpha}\right]-1}, \quad x \geq k .
$$

We obtain the Pareto distribution as a sub-model when $\theta \rightarrow 0$. Figures 3 and 4 display the density and hazard rate functions of the CMWG distribution for selected parameter values.

The $r$ th moment of the CPP random variable becomes

$\mathrm{E}\left(X^{r}\right)=\alpha k^{r} \sum_{n=1}^{\infty} \sum_{j=0}^{n-1} \frac{(j+1)}{(j+1) \alpha-r} \omega_{n, j}(\theta), \quad(j+1) \alpha>r$.

In particular, setting $r=1$ in 20, we obtain the mean of $X$

$$
\mathrm{E}(X)=\alpha k \sum_{n=1}^{\infty} \sum_{j=0}^{n-1} \frac{(j+1)}{(j+1) \alpha-1} \omega_{n, j}(\theta), \quad(j+1) \alpha>1 .
$$

\section{Applications}

In this section, we compare the fits of some special models of the CEWPS class by means of a real data set to show its potentiality. In order to estimate the parameters of these special models, we adopt the maximum likelihood method (as discussed in Section 4). All the computations were done using the subroutine NLMixed of the SAS software.

The first data set consists of a real data set obtained from Smith and Naylor (1987). The data are the strengths of $1.5 \mathrm{~cm}$ glass fibres, measured at the National Physical Laboratory, England. The data are: 0.55, 0.74, 0.77, 0.81, $0.84,1.24,0.93,1.04,1.11,1.13,1.30,1.25,1.27,1.28$, $1.29,1.48,1.36,1.39,1.42,1.48,1.51,1.49,1.49,1.50,1.50$, $1.55,1.52,1.53,1.54,1.55,1.61,1.58,1.59,1.60,1.61,1.63$, $1.61,1.61,1.62,1.62,1.67,1.64,1.66,1.66,1.66,1.70,1.68$, $1.68,1.69,1.70,1.78,1.73,1.76,1.76,1.77,1.89,1.81,1.82$, $1.84,1.84,2.00,2.01,2.24$. Table 4 summarizes some descriptives statistics about the current data.

Table 4: Descriptive statistics for the strengths of glass fibres data.

\begin{tabular}{cccccc}
\hline Min & 1st Q & Median & Mean & 3rd Q & Max \\
\hline 0.550 & 1.375 & 1.590 & 1.507 & 1.685 & 2.240 \\
\hline
\end{tabular}

The CEWPS model in the application is defined by equation (5) with $H(x ; \xi)=x^{\gamma}, h(x ; \xi)=\gamma x^{\gamma-1}$ and $C(\theta)=\theta(1-\theta)^{-1}$, thus referring to the complementary Weibull geometric (CWG) distribution with $\boldsymbol{\theta}_{\mathbf{1}}=$ $(\alpha, \gamma, \theta)$. For comparison purposes, we compare the CWG distribution with the Weibull, modified Weibull (MW), complementary exponentiated exponential geometric (CEEG) and complementary exponential power (CEP) models defined, respectively, as

$$
\begin{aligned}
f\left(x ; \boldsymbol{\theta}_{2}\right) & =\alpha \beta^{\alpha} x^{\alpha-1} \exp \left\{-(\beta x)^{\alpha}\right\}, \\
f\left(x ; \boldsymbol{\theta}_{3}\right) & =\alpha(\beta+\lambda x) x^{\beta-1} \exp \left(\lambda x-\alpha x^{\beta} \mathrm{e}^{\lambda x}\right), \\
f\left(x ; \boldsymbol{\theta}_{4}\right) & =\alpha \theta \lambda \mathrm{e}^{-\lambda x}\left(1-\mathrm{e}^{-\lambda x}\right)^{\alpha-1} \\
& {\left[1-(1-\theta)\left(1-\mathrm{e}^{-\lambda x}\right)^{\alpha}\right]^{-2} }
\end{aligned}
$$

and

$$
\begin{gathered}
f\left(x ; \boldsymbol{\theta}_{5}\right)=\frac{\beta \theta x^{\beta-1}}{\alpha^{\beta}} \exp \left\{1+\left(\frac{x}{\alpha}\right)^{\beta}-\exp \left[\left(\frac{x}{\alpha}\right)^{\beta}\right]\right\} \\
\left\{\left[1-\exp \left(1-\exp \left[\left(\frac{x}{\alpha}\right)^{\beta}\right]\right)\right\}^{\theta-1},\right.
\end{gathered}
$$

for $x>0$, where $\boldsymbol{\theta}_{2}=(\alpha, \beta), \boldsymbol{\theta}_{3}=(\alpha, \lambda, \beta), \boldsymbol{\theta}_{4}=(\alpha, \lambda, \theta)$ and $\theta_{5}=(\alpha, \beta, \theta)$.

The MLEs of the parameters (with standards errors), the $-2 \ell(\widehat{\boldsymbol{\Theta}})$ and Kolmogorov-Smirnov (K-S) statistics are given in Table 5 . From those statistics, we conclude that the CWG model provides a better fit to the data set, since it yields the lowest values for these statistics.

We also perform formal goodness-of-fit tests in order to verify which distribution fits better to the data. We apply the Cramér-von Mises (C-M) and ArdersonDarling $(A-D)$ tests. The C-M and A-D test statistics are described in details in Chen and Balakrishnan (1995). 


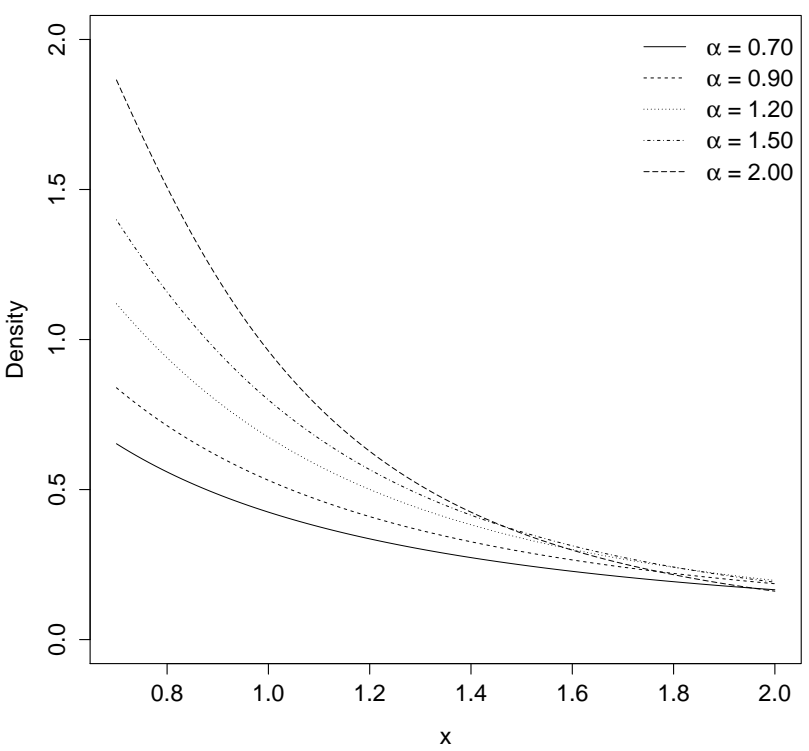

(a) $\theta=0.8$ and $k=0.7$

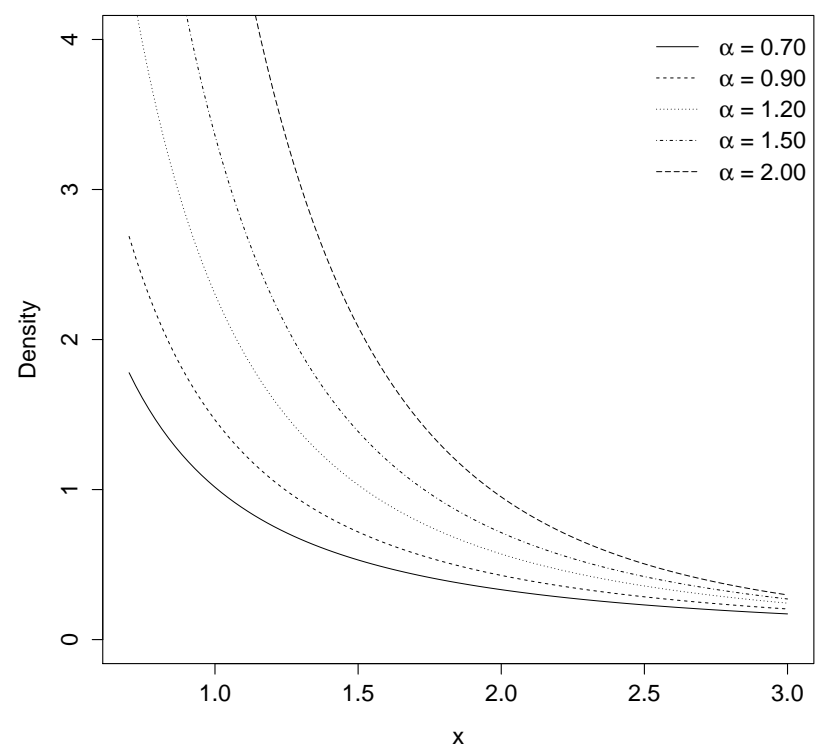

(b) $\theta=0.1$ and $k=2$

Figure 3: Plots of the CPP densities for some parameter values.

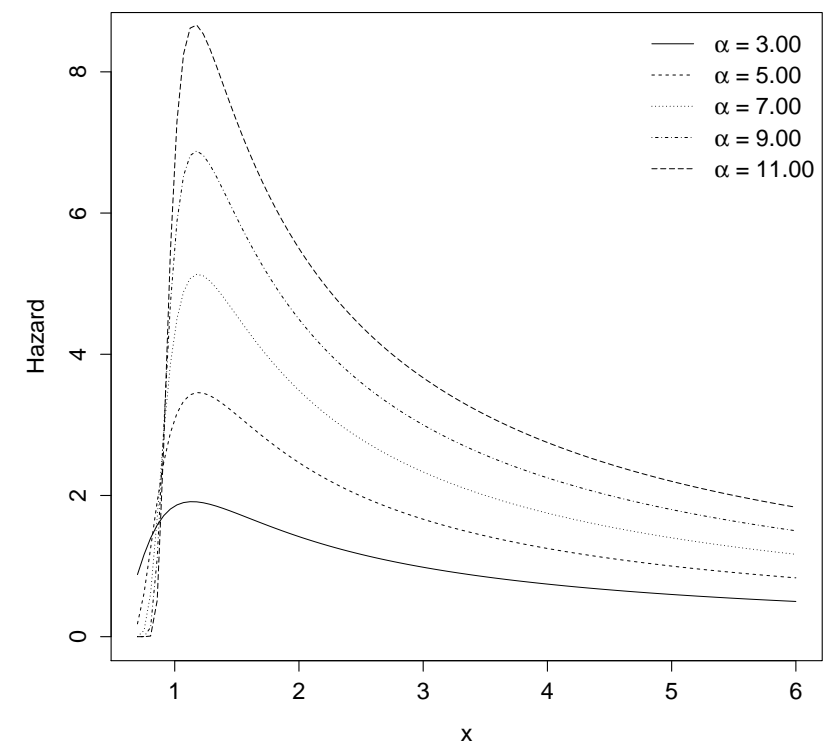

(a) $\theta=0.9$ and $k=1$

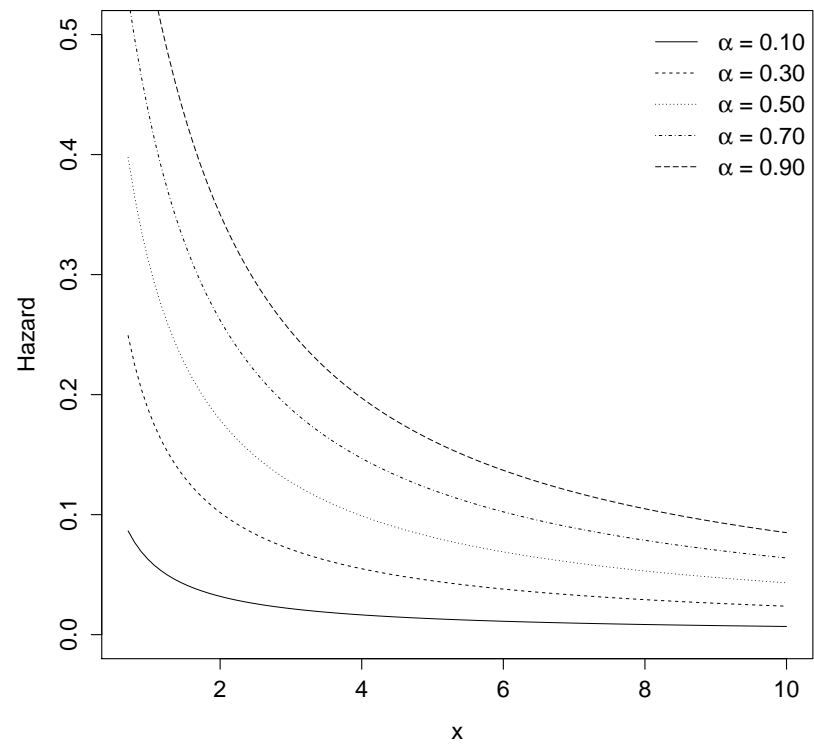

(b) $\theta=0.8$ and $k=1$

Figure 4: Plots of the CPP hrfs for some parameter values.

In general, the smaller the values of C-M and A-D, the better the fit to the data. Table 5 gives the values of these four statistics for the data. According to them, the CWG model fits the data set better than the others competing models.

Plots of the pdfs and cdfs of the fitted CWG, Weibull, MW, CEEG and CEP models to the data, displayed in
Figure 5, indicate the superiority of the CWG model.

\section{Concluding remarks}

We define a new class of lifetime distributions named the complementary extended Weibull power series (CEWPS) class on a latent complementary risk problem base. The 
Table 5: Estimates ( ${ }^{a}$ denotes standard errors) and $-2 \ell(\widehat{\boldsymbol{\Theta}})$, A-D, C-M and K-S statistics $\left({ }^{b}\right.$ denotes p-values)

\begin{tabular}{|c|c|c|c|c|c|}
\hline Distribution & Estimates & $-2 \ell(\widehat{\boldsymbol{\Theta}})$ & A-D & C-M & K-S \\
\hline CWG & $\begin{aligned} \widehat{\boldsymbol{\theta}}_{\mathbf{1}}= & (0.6948,3.2018,0.9399) \\
& (0.5651,0.9478,0.0750)^{a}\end{aligned}$ & 24.1 & $\begin{array}{c}0.5839 \\
(0.1231)^{b}\end{array}$ & $\begin{array}{c}0.1049 \\
(0.0940)\end{array}$ & $\begin{array}{c}0.1000 \\
(0.5542)\end{array}$ \\
\hline Weibull & $\begin{aligned} \widehat{\theta}_{2}= & (5.7807,0.6142) \\
& (0.5761,0.0140)^{a}\end{aligned}$ & 30.4 & $\begin{array}{c}1.2877 \\
(0.0022)^{b}\end{array}$ & $\begin{array}{c}0.2324 \\
(0.0018)\end{array}$ & $\begin{array}{c}0.1522 \\
(0.1079)\end{array}$ \\
\hline MW & $\begin{aligned} \widehat{\theta}_{3}= & (0.0087,2.1608,2.4027) \\
& (0.0126,1.5418,2.4173)^{a}\end{aligned}$ & 28.7 & $\begin{array}{c}0.9497 \\
(0.0153)^{b}\end{array}$ & $\begin{array}{c}0.1695 \\
(0.0127)\end{array}$ & $\begin{array}{c}0.1352 \\
(0.1995)\end{array}$ \\
\hline CEEG & $\begin{aligned} \widehat{\theta}_{4}= & (10.9697,5.8364,0.0014) \\
& (58.0308,0.6471,0.0075)^{a}\end{aligned}$ & 32.0 & $\begin{array}{c}1.4550 \\
(0.0008)^{b}\end{array}$ & $\begin{array}{c}0.2658 \\
(0.0008)\end{array}$ & $\begin{array}{c}0.1326 \\
(0.2178)\end{array}$ \\
\hline CEP & $\begin{aligned} \widehat{\theta}_{5}= & (1.6932,2.7908,1.7899) \\
& (0.0961,0.6707,0.6947)^{a}\end{aligned}$ & 30.0 & $\begin{array}{c}1.1638 \\
(0.0045)^{b}\end{array}$ & $\begin{array}{c}0.2103 \\
(0.0038)\end{array}$ & $\begin{array}{c}0.1485 \\
(0.1240)\end{array}$ \\
\hline
\end{tabular}

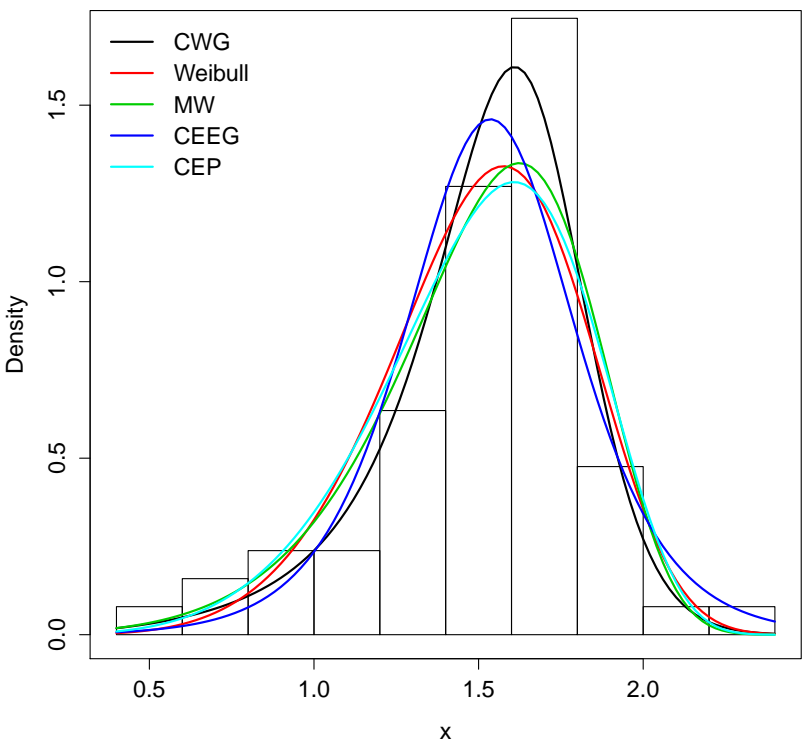

(a)

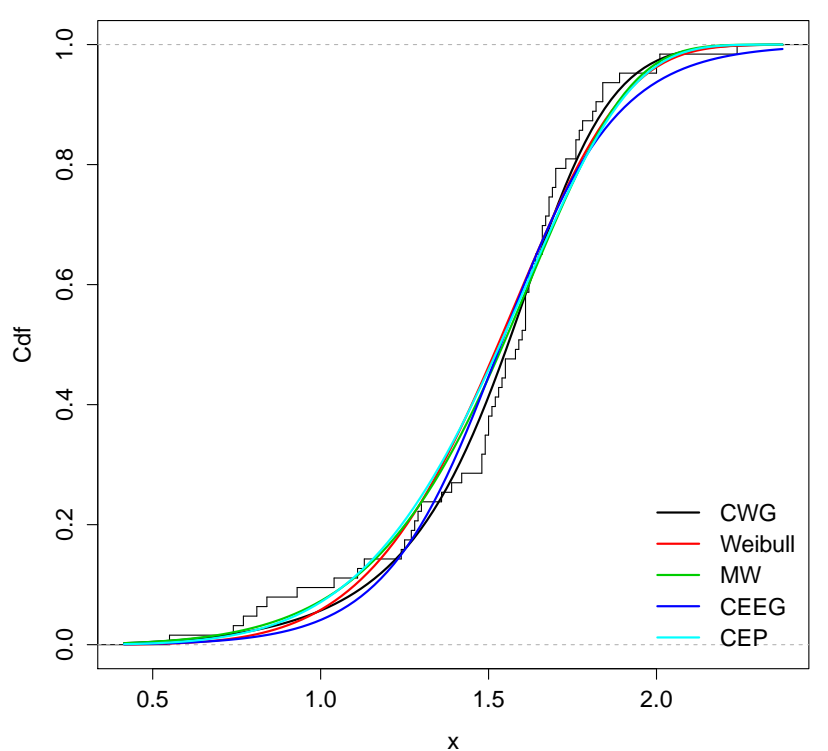

(b)

Figure 5: Estimated (a) pdfs and (b) cdfs for the CWG, Weibull, MW, CEEG and CEP models for the strengths of glass fibres data.

new class extends several models existing in literature such as the complementary exponential geometric distribution (Louzada et al., 2011), the Poisson exponential distribution (Cancho et al., 2011) and complementary exponential power series class (Flores et al., 2013). We provide a mathematical treatment of the new class including expansions for the density function, ordinary and incomplete moments, and generating function. The CEWPS density function can be expressed as a linear combination of extended Weibull (EW) density functions. This linear combination representation is important to derive several properties of the new class. Maximum likelihood inference is implemented straightforwardly for estimating the model parameters. Some special models are explored. We fit some CEWPS distributions to a real data set to show the usefulness of the proposed class. In conclusion: we define a general approach for generating new lifetime distributions, at least 68 distributions, some of them known and the great majority new ones. Further, we motivate the use of the new class in two different ways. We think these two facts combined may attract more complex applications in the literature of lifetime distributions. Finally, the formulas derived are manageable by using modern computer resources with analytic and numerical capabilities. 


\section{Acknowledgments}

The authors gratefully acknowledge financial support from CAPES (Brazil) and CNPq (Brazil).

\section{References}

Barriga, G.D.C., Louzada-Neto, F., Cancho, V.G. (2011). The complementary exponential power lifetime model. Computational Statistics and Data Analysis, 55, 1250-1259.

Bebbington, M., Lai, C.D., Zitikis, R. (2007). A flexible Weibull extension. Reliability Engineering and System Safety, 92, 719-726.

Bereta, M.P., Louzada-Neto, F., Franco, M.A.P. (2010). A distribuição Weibull-Poisson, SINAPE - Simpósio Nacional de Probabilidade e Estatística, 19, São Pedro-SP.

Cancho, V.G., Louzada, F., Barriga. G.D.C. (2011). The Poisson-exponential lifetime distribution. Computational Statistics and Data Analysis, 55, 677-686.

Carrasco J.M.F., Ortega, E.M.M., Cordeiro, G.M. (2008). A generalized modified Weibull distribution for lifetime modeling. Computational Statistics and Data Analysis, 53, 450-462.

Chen, Z. (2000). A new two-parameter lifetime distribution with bathtub shape or increasing failure rate function. Statistics and Probability Letters, 49, 155-161.

Cox, D.R., Hinkley, D.V. (1974). Theoretical Statistics. Chapman and Hall, London.

Cox, D.R., Oakes, D. (1984). Analysis of Survival Data. London: Chapman and Hall.

Crowder, M.J., Kimber, A.C., Smith, R.L., Sweeting, T.J. (1991). Statistical Analysis of Reliability Data. Chapman \& Hall, London.

Doornik, J. (2007) Ox 5: object-oriented matrix programming language, 5th ed. Timberlake Consultants, London.

Flores, J.D., Borges, P., Cancho, V.G., Louzada, F. (2013). The complementary exponential power series distributions. Brazilian Journal of Probability and Statistics, 4, 565-584.

Gompertz, B. (1825). On the nature of the function expressive of the law of human mortality and on the new model of determining the value of life contingencies. Philosophical Trans. Royal Society of London, 115, 513-585.
Guess F., Proschan F. (1985). Mean residual life: theory and applications. In P.R. Krishnaiah and C.R. Rao (eds.) Handbook of Statistics. Reliability and Qualiy Control, 7, 215-224.

Gurvich, M., DiBenedetto, A., Ranade, S. (1997). A new statistical distribution for characterizing the random strength of brittle materials. Journal of $\mathrm{Ma}$ terials Science, 32, 2559-2564.

Johnson, N.L, Kotz, S., Balakrishnan, N. (1994). Continuous Univariate Distributions volume 1. John Wiley \& Sons, New York.

Kies, J.A. (1958). The strength of glass. Washington D.C. Naval Research Lab, 5093.

Lai, C.D., Xie, M., Murthy, D.N.P. (2003). A modified Weibull distribution. Transactions on Reliability, 52, 33-37.

Lawless J.F. (2003) Statistical models and methods for lifetime data. John Wiley and Sons, 2nd edition.

Louzada-Neto, F. (1999). Poly-hazard regression models for lifetime data. Biometrics, 55, 1121-1125.

Louzada, F., Marchi, R., Carpenter, J. (2013). The Complementary Exponentiated Exponential Geometric Lifetime Distribution. Journal of Probability and Statistics, http://dx.doi .org/10.1155/2013/502159.

Louzada, F., Roman, M., Cancho, V.G. (2011). The complementary exponential geometric distribution: Model, properties, and a comparison with its counterpart. Computational Statistics and Data Analysis, 55, 2516-2524.

Marshall, A.W., Olkin, I. (1997). A new method for adding a parameter to a family of distributions with application to exponential and Weibull families. Biometrika, 84, 641-652.

Morais, A.L., Barreto-Souza, W. (2011). A Compound Class of Weibull and Power Series Distributions. Computational Statistics and Data Analysis, 55, 14101425.

Nadarajah, S., Kotz, S. (2005). On some recent modifications of Weibull distribution. IEEE Trans. Reliability, 54, 561-562.

Nikulin, M., Haghighi, F. (2006). A Chi-squared test for the generalized power Weibull family for the head-and-neck cancer censored data. Journal of Mathematical Sciences, 133, 1333-1341.

Pham, H. (2002). A vtub-shaped hazard rate function with applications to system safety. International Journal of Reliability and Applications, 3, 1-16. 
Pham, H., Lai, C-D. (2007). On recent generalizations of the Weibull Distribution. IEEE Transactions on Reliabilty, 56, 454-458.

Phani, K.K. (1987). A new modified Weibull distribution function. Communications of the American Ceramic Society, 70, 182-184.

Prudnikov, A.P., Brychkov, Yu.A., Marichev, O. I. (1986). Integrals and Series, Vol. 1: Elementary Functions. New York: Gordon and Breach.

R Development Core Team, 2009. R: A Language and Environment for Statistical Computing. Vienna, Austria.

Rayleigh, J.W.S. (1880). On the resultant of a large number of vibrations of the same pitch and of arbitrary phase. Philosophical Magazine, 10, 73-78.

Sigmon, K., Davis, T.A., 2002. MATLAB Primer, 6th ed. Chapman and Hall/CRC, London.

Silva, R.B., Bourguignon, M., Dias, C.R.B., Cordeiro, G.M. (2013). The compound class of extended Weibull power series distributions. Computational Statistics and Data Analysis, 58, 352-367.

Smith, R.M., Bain, L.J. (1975). An exponential power life-testing distribution. Communications Statistics, 4, 469-481.

Smith, R.L., Naylor, J. (1987) A comparison of maximum likelihood and Bayesian estimators for the three-parameter Weibull distribution. Applied Statistics, 36, 358-369.

Xie, M., Lai, C.D. (1995). Reliability analysis using additive Weibull model with bathtub-shaped failure rate function. Reliability Engineering and System Safety, 52, 87-93.

Xie, M., Tang, Y., Goh, T.N. (2002). A modified Weibull extension with bathtub-shaped failure rate function. Reliability Engineering and System Safety, 76, 279-285.

White, J. S. (1969). The moments of log-Weibull order statistics. Technometrics, 11, 373-386.

Wolfram, S. (2003). The Mathematica Book, 5th ed. Cambridge University Press. 